\title{
Therapeutic Effect of Combined Dabrafenib and Trametinib Treatment of BRAF V600E-Mutated Primary Squamous Cell Carcinoma of the Thyroid: A Case Report
}

\author{
Tim Brandenburg ${ }^{a, b} \quad$ Philipp Muchalla ${ }^{a, b}$ Sarah Theurer ${ }^{b, c}$ \\ Kurt Werner Schmid ${ }^{b, c}$ Dagmar Führer ${ }^{a, b}$
}

aDepartment of Endocrinology, Diabetes and Metabolism, Endocrine Tumour Center at West German Cancer

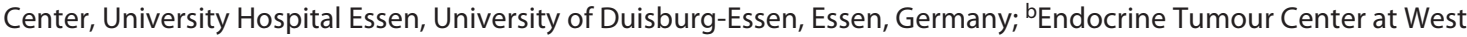
German Cancer Center, Member of ENDO-ERN and EURACAN, University Hospital Essen, University of DuisburgEssen, Essen, Germany; ' University Duisburg-Essen, Institute of Pathology, University Hospital Essen, University of Duisburg-Essen, Essen, Germany

\section{Established Facts}

- Primary squamous cell carcinoma (PSCC) of the thyroid is an orphan disease and is usually diagnosed at an advanced stage causing compressive symptoms.

- Median survival after diagnosis is comparable to the survival of anaplastic thyroid carcinoma (ATC).

- No established therapy is available.

\section{Novel Insights}

- Molecular pathology revealed a therapeutic target (BRAF V600E mutation), which was effectively treated with combined BRAF/MEK inhibition by dabrafenib and trametinib for a period of $>12$ months with drastic improvement in patient's quality of life and without severe side effects.

\section{Keywords}

Primary squamous cell carcinoma of the thyroid $\cdot$ BRAF

V600E $\cdot$ Dabrafenib · Trametinib

\section{Abstract \\ Introduction: Primary squamous cell carcinoma (PSCC) of the thyroid is an exceptionally rare malignancy accounting for $<1 \%$ of all primary thyroid cancers. Therapy is multimod- al including surgery, radiotherapy, and chemotherapy but}

with no consensus for management and therapy. Here, we describe a case of a male patient who presented with a BRAF V600E-mutated PSCC of the thyroid gland showing response to combined dabrafenib and trametinib therapy over a period of >12 months. Case Presentation: A 78-year-old male patient presented with a 3-week history of dysphonia and dyspnoea. Laryngoscopy revealed a mechanical obstruction by a right-sided, subglottical mass, which on cervical ultrasound was highly suggestive of anaplastic thyroid carcinoma. Additional workup including esophagogastroduode-
Correspondence to:

Tim Brandenburg, tim.brandenburg@uk-essen.de 
noscopy showed compression of the oesophagus but no oesophageal infiltration by the tumour. Immunohistochemistry displayed CK19-positive cells indicating epithelial origin of the tumour. CK5/6 and P40 immunohistochemistry confirmed the morphological impression of squamous cell differentiation while staining with thyroid markers TTF- 1 and TPO was negative and PAX8 showed a nuclear positive signal. Based on immunohistopathology, presence of TP53 and BRAF V600E mutations, and exclusion of metastatic squamous cell carcinoma of other origin, the diagnosis of a PSCC of the thyroid was established. As an individualized treatment concept, we decided to advocate combined BRAF V600E targeting by the multikinase inhibitors dabrafenib and trametinib. This led to drastic improvement in patient's quality of life without severe side effects over a period of $>12$ months. Conclusion: In this case, molecular diagnosis allowed a highly individualized treatment concept with combined dabrafenib and trametinib therapy.

(c) 2021 European Thyroid Association Published by S. Karger AG, Basel

\section{Introduction}

Primary squamous cell carcinoma (PSCC) of the thyroid accounts for $<1 \%$ of all primary thyroid malignancies [1-6]. PSCC is usually diagnosed at an advanced stage causing compressive neck symptoms like dysphagia and dyspnoea. Median age at presentation is in the fifth till seventh decade of life [3,5-7], and the median survival after diagnosis is comparable to anaplastic thyroid carcinoma (ATC) $[3,6,7]$. Before diagnosis, infiltration or metastasizing from other primary focuses of squamous cell carcinoma to the thyroid gland must be excluded [4-6]. Referring to the current 4th edition of the World Health Organization classification for thyroid tumours [8], squamous cell carcinoma of the thyroid should be composed predominantly or entirely of tumour cells with squamous differentiation resulting in a positive PAX8 staining. The latter is important to differentiate primary from secondary squamous cell carcinomas. Additionally, by definition, PSCC should harbour a TP53 mutation. Since PAX8 is still expressed in about $50 \%$ of ATCs showing a similar clinical presentation and with TP53 being the most frequently mutated gene also in ATC [8], the differential diagnosis may be challenging. However, as established in ATC, tumours derived from thyroid tissue can retain driver mutations such as BRAF V600E [3, 9-11]. Based on the results of a basket trial, the Food and Drug Administration has recently approved the combined application of dabrafenib (selective BRAF inhibitor) and trametinib (selective MEK inhibitor) for BRAF V600E-mutated ATC [12]. Out of the enrolled 16 patients, one experienced a complete response, and 10 patients showed partial responses under combined dabrafenib and trametinib therapy. Among the responders, 9 patients had no significant tumour growth over at least 6 months [12]. We describe a case of a patient with an initially unresectable BRAF V600E-mutated thyroid PSCC, who was effectively treated with dabrafenib and trametinib over a period of 12 months with drastic improvement in quality of life before developing drug resistance.

\section{Case Presentation}

A 78-year-old male patient presented to his primary physician with a 3-week history of dysphonia and dyspnoea. He was an exsmoker (35 py, nicotine abuse for 27 years), COPD Gold II, and was fully anticoagulated due to a mechanic aortic heart valve implantation 19 years ago. Laryngoscopy revealed a mechanical obstruction by a right-sided, subglottical mass. Baseline FDG-PETCT showed a 5.4-cm (SUV max. 10.3) lesion in the right thyroid lobe (shown in Fig. 1) causing tracheal deviation and compression. No significant lymphadenopathy was found. On cervical ultrasound, the lesion was highly suggestive of ATC. Due to rapidly progressing airway obstruction, emergency tracheostomy was performed along with a tumour biopsy. Initial pathology revealed a poorly differentiated thyroid carcinoma. Additional workup including esophagogastroduodenoscopy (EGD) showed compression of the oesophagus but no oesophageal infiltration by the tumour. A percutaneous endoscopic gastrostomy tube was installed to secure nutrition. The patient underwent fractionated intensitymodulated radiotherapy $(10 \times 3.5 \mathrm{~Gy})$ over a period of 2 weeks, before presenting to our department for further evaluation of treatment. At first presentation, the patient complained of dysphonia and dysphagia, and swallowing of food was hardly possible and accompanied by severe pain and malaise. No further dyspnoea was reported (70 kg, BMI $22.5 \mathrm{~kg} / \mathrm{m}^{2}$, ECOG 1). Re-evaluation of the histology (fresh biopsies and archival biopsies) (shown in Fig. 2) revealed a PSCC, possibly of thyroid origin. Immunohistochemistry (shown in Fig. 2b) displayed CK19-positive tumour cells indicating epithelial origin of the mass. CK5/6 and P40 immunohistochemistry confirmed the histomorphological impression of squamous cell differentiation (shown in Fig. 2c, d). Staining with thyroid markers TTF-1 and TPO was negative (shown in Fig. 2e, f), while PAX8 staining was positive (shown in Fig. $2 \mathrm{~g}$ ). Molecular diagnostics revealed a TP53 and a BRAF V600E mutation in the tumour tissue (shown in Table 1), TERT promoter status was not analysed, and re-evaluation of archival tumour material for TERT status 3 years later was not successful. Additionally, an FDG-PETCT was performed (shown in Fig. 3a) showing a pulmonary lesion of $31 \times 24 \mathrm{~mm}$ (SUV max 1.6), which had been known for 14 years but no other extrathyroidal lesions. Due to the anticoagulation and the patient's condition, biopsy of the pulmonary lesion was not performed. 


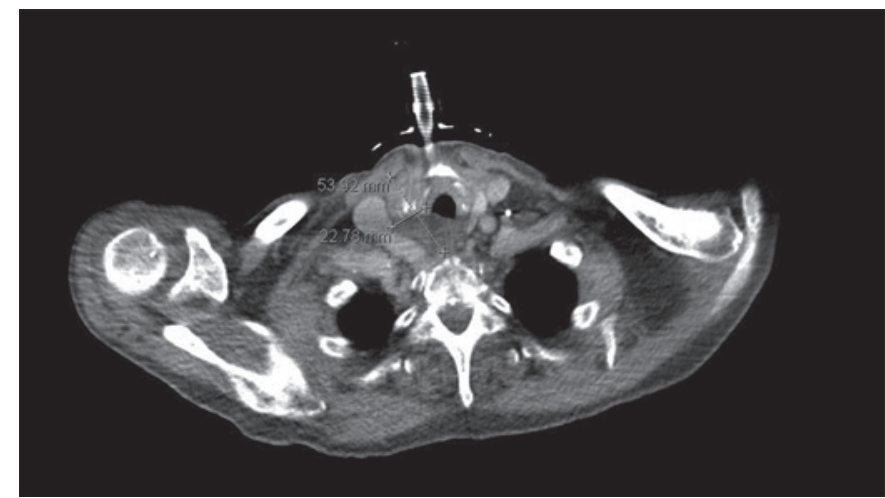

Fig. 1. FDG-PET-CT performed on 09/17 before treatment start showing a $5.4 \times 2.3 \mathrm{~cm}$ mass in the right thyroid gland causing lateral displacement of the trachea, the common carotid artery, and the internal jugular vein, which corresponded to an area with enhanced PET tracer uptake (SUV max. 10.3).

Based on immunohistopathology, molecular diagnostics, and exclusion of metastatic squamous cell carcinoma of other origin by FDG-PET-CT, EGD, and laryngoscopy, the diagnosis of a PSCC of the thyroid was established in the patient. Due to the limited known therapeutic options, we decided to advocate combined BRAF V600E/MEK targeting by multikinase inhibitors (MKI) dabrafenib and trametinib [13] as an individualized treatment concept, which was funded by the patient's health insurance company as an unlicensed indication.

Before MKI therapy start, the tracheostoma was removed, and a tracheal stent was inserted. A first follow-up imaging on MKI treatment was performed at 3 months by FDG-PET-CT and showed a partial remission (shown in Fig. 3b). Besides fatigue (CTCAE 1) and an episode of drug-induced pyrexia shortly after therapy initiation, no severe side effects were observed, and the therapy was well tolerated. Consumption of smooth food and liquids was again possible (ECOG 1) without malaise leading to substantial weight gain (BMI $23 \mathrm{~kg} / \mathrm{m}^{2}$ ). At 5 months after MKI start, the percutaneous endoscopic gastrostomy tube and the inlaying tracheal stent were removed leading to immense improvement in well-being of the patient. Imaging at 6 months confirmed stable disease (shown in Fig. 3c). At 9.5 months (shown in Fig. 3d), a small regrowth in tumour size was noted. The pulmonary lesion remained stable, but a suspicious mediastinal lymph node of $6 \mathrm{~mm}$ (SUV max. 11.4) was detected. Considering the initial tumour mass and the so far welltolerated therapy, MKI treatment was continued. At 13 months (shown in Fig. 3e), imaging revealed a further local progress, still less compared to the original finding. The lung lesion remained stable, but the previously detected mediastinal lymph node gained size (14 $\mathrm{mm}$, before $6 \mathrm{~mm}$ ). The patient's weight was stable (BMI $23.2 \mathrm{~kg} /$ $\mathrm{m}^{2}$ ). There was no dyspnoea, but eventually dysphagia occurred. EGD was performed showing no tumour infiltration but a scarred stenosis which was mechanically dilatated. At 16 months (shown in Fig. 3f), progression was obvious with the primary tumour reaching its pre-treatment size and compression of the trachea leading to dyspnoea. Substantial tumour infiltration of the larynx, trachea, and oesophagus was noted. On CT scan, multiple cervical, paratracheal, and mediastinal lymph node metastasis were found, while the lung
Table 1. Molecular analysis by NGS in tumour biopsy

\begin{tabular}{lll}
\hline Gens & Exons & DNA level \\
\hline BRAF & 15 & c. 199T>A \\
& 11 & Wildtype \\
DDR2 & $15,16,17,18$ & Wildtype \\
EGFR & $18,19,20,21$ & Wildtype \\
ERBB2 & $5,6,15,20,23,29$ & Wildtype \\
FGFR1 & $3,7,13,17$ & Wildtype \\
FGFR3 & 7,9 & Wildtype \\
HRAS & $2,3,4$ & Wildtype \\
KIT & $9,10,11,13,17,18$ & Wildtype \\
KRAS & $2,3,4$ & Wildtype \\
MET & $3,8,11,14,19$ & Wildtype \\
NRAS & $2,3,4$ & Wildtype \\
PDGFRa & $12,14,18$ & Wildtype \\
PIK3CA & $3,5,10,16,21$ & Wildtype \\
RET & $10,11,13,14,15,16$ & Wildtype \\
TP53 & 5 & c. 524 G $>$ A \\
& $4,6,7,8,9$ & Wildtype \\
\hline
\end{tabular}

NGS, next-generation sequencing.

lesion remained stable. Due to the massive tumour progress along with a deterioration of the patient's condition due to dysphagia and dyspnoea (ECOG 3-4) as well as beginning of dementia, a decision was made with the family to stop treatment in the 80 -year-old patient. The patient died 1 month later, 20 months after first diagnosis of the PSCC.

\section{Discussion}

This is the first report of combined long-term dabrafenib and trametinib usage for the treatment of a BRAF V600E-positive PSCC of the thyroid [14]. Two critical points are accentuated: patients carrying a drug targetable mutation in an orphan cancer must be identified since they may benefit from targeted treatment as also shown for, for example, BRAF V600E-positive ATC $[12,15]$. According to the literature, only 2 BRAF V600E-mutated PSCCs $[14,16]$ have previously been described (one simultaneously while preparing this manuscript) along with 4 other BRAF V600E-mutated cases of PTC with coexisting squamous carcinoma components [3, 17-20]. The reported average median survival in PSCC of the thyroid of 6-8 months $[3,6,7]$ was exceeded in our patient who presented with life-threatening dyspnoea and dysphagia at onset of disease, but experienced impressive symptom control under combined MKI treatment resulting in a good quality of life for at least 12 months. Although contribution of radiotherapy cannot be excluded, 


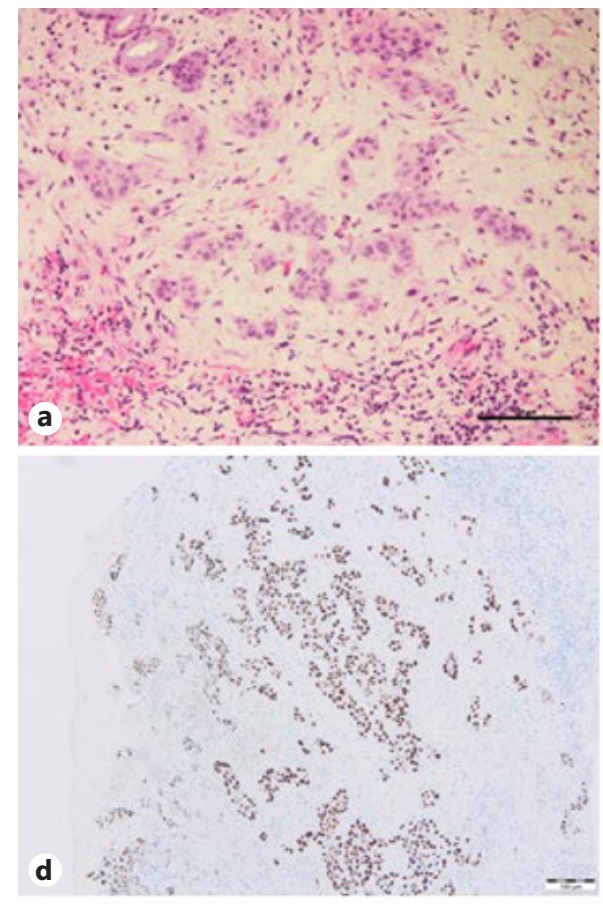

g

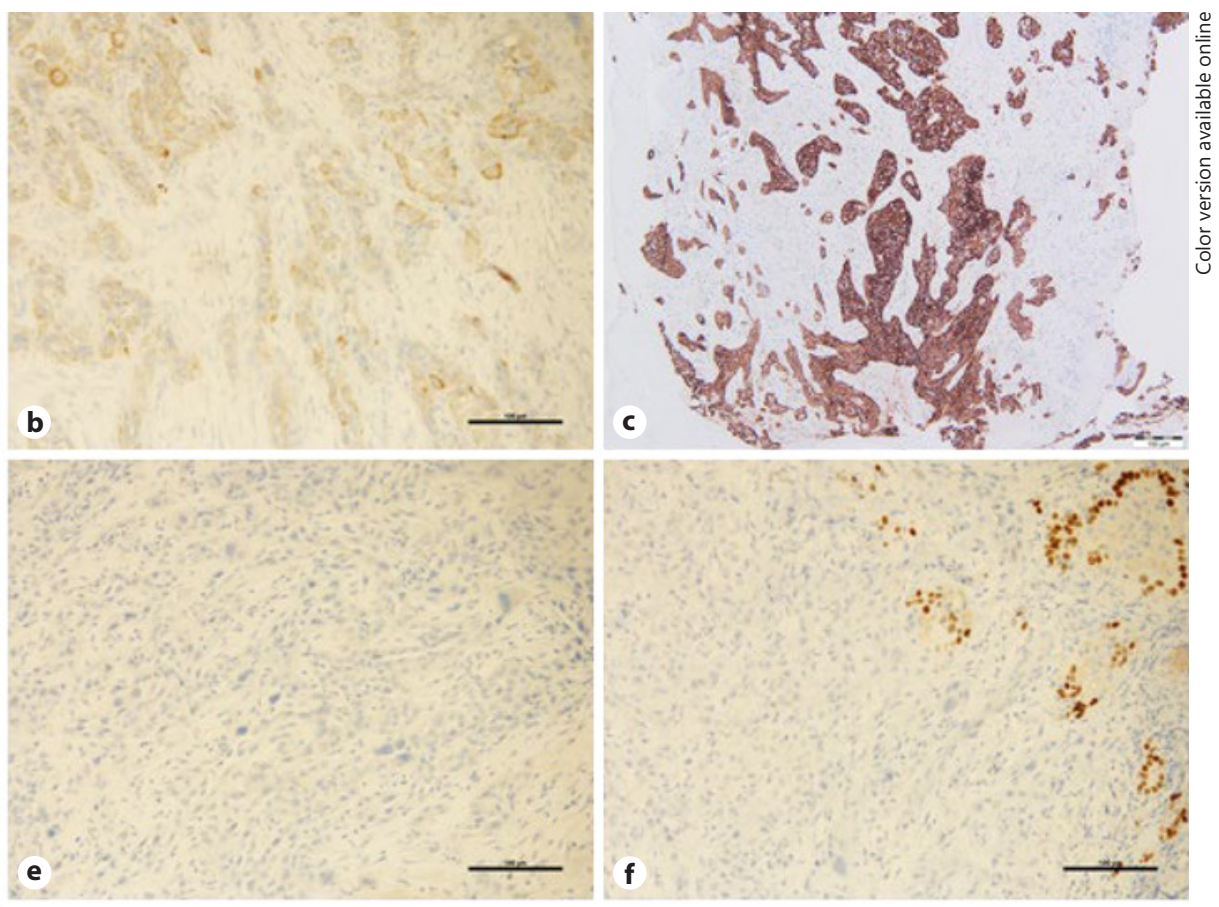

Fig. 2. a Haematoxylin and eosin staining showing squamous cell nests in a representative tumour biopsy. b Immunofluorescence of the tumour biopsy displayed CK19-positive cells indicating epithelial origin. CK5 and 6 (c) and P40 (d) positivity confirmed morphological impression of squamous cell differentiation. TTF-1 (e) and TPO (f) (thyroid marker) staining was negative. g PAX8 staining showed a nuclear expression pattern. Scale bar, $100 \mu \mathrm{m}$. the massive reduction in tumour size 3 months after start of dabrafenib and trametinib is most likely the result of combined MKI therapy $[12,15]$. Second, drug resistance evolved slowly with a first small new lesion occurring at 9.5 months after treatment initiation and then gained speed resulting in rapid tumour progression at 16 months. Montero-Conde et al. [21] demonstrated a rebound in ERK signalling using the BRAF inhibitor vemurafinib in thyroid cancer cell lines, which was associated with HER2/ HER3 expression. Similar mechanisms might occur upon dabrafenib and trametinib usage. Acquired bypass pathways such as in BRAF inhibitor-treated melanoma are another possibility. According to Wagle et al. [22], these could be gained by overexpression of COT or PDGFRbeta, which in turn may reactivate MAPK signalling. Taken together, to fully understand the underlying mechanism of MKI resistance in this case, translational studies are needed. As dementia symptoms were noted in the 80-year-old patient, best supportive care was offered instead of a switch in therapy. CT imaging at this stage revealed no cerebral lesion. The patient succumbed to the tumour about 20 months after first diagnosis. It remains an open question whether our patient might have benefited from additional immunotherapy. For instance, ATC tumours have been shown to express PD-L1 [15, 23, 24], and improved response to checkpoint inhibition has been demonstrated in a recent trial using the PD-1 inhibitor spartalizumab [25]. Retrospective analysis revealed only weak PD-L1 expression (2\%) in the biopsy sample. In summary, in this case, re-evaluation and molecular analysis allowed an individualized treatment concept using combined dabrafenib and trametinib treatment with a substantial therapeutic effect in a very rare BRAF V600Emutated PSCC of the thyroid. 

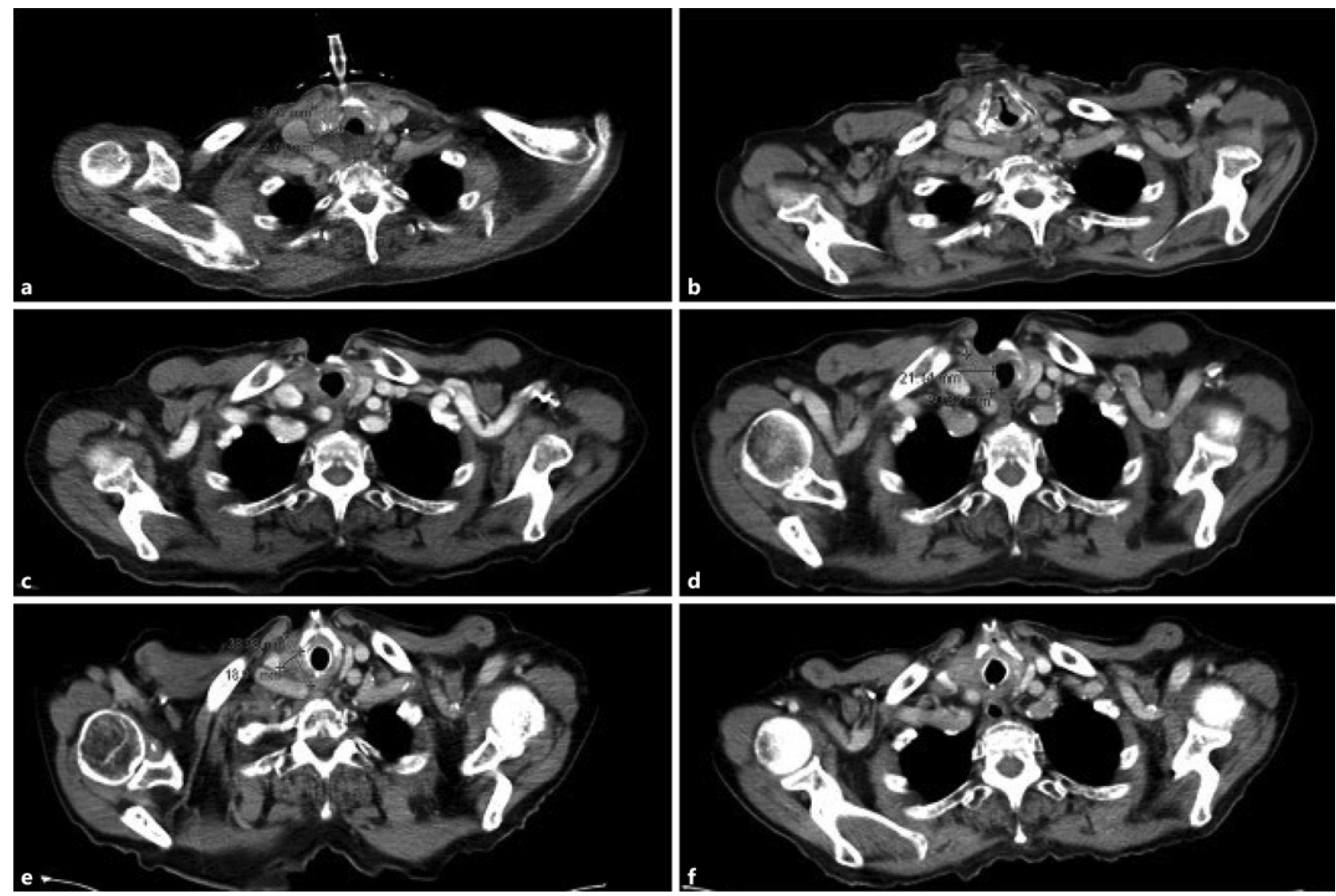

Fig. 3. Representative neck CT scans showing response of the PSCC of the thyroid to combined MKI treatment (baseline, a) at 3 (b) and 6 (c) months and regrowth at $9.5(\mathbf{d}), 13(\mathbf{e})$, and 16 (f) months. PSCC, primary squamous cell carcinoma.

\section{Statement of Ethics}

Written informed consent was obtained from the patient's next of kin for publication of this case report and any accompanying images.

\section{Conflict of Interest Statement}

T.B. received honoraria and/or expenses for invited speeches from Eisai, Lilly, Liberum, and Bayer Pharma. P.M. received honoraria and/or expenses for invited speeches from Eisai. S.T. and K.W.S. have no conflicts of interest to declare. D.F. received honoraria and/or expenses for invited speeches from Eisai, Ipsen, and Sanofi Genzyme and is a member of the advisory board of Eisai, Ipsen, and Sanofi Genzyme.

\section{Funding Sources}

This research received no specific funding.

\section{Author Contributions}

T.B. organized and designed the figure, wrote the manuscript, and was involved in primary patient's care. P.M. was involved in primary patient's care, data analysis, and manuscript correction. S.T. and K.W.S. provided reagents, performed and analysed histology, discussed the data, and corrected the manuscript. D.F. initiated the case report, was involved in patient care, designed, and corrected the manuscript.

\section{References}

Eur Thyroid J 2021;10:511-516 DOI: $10.1159 / 000518055$
MKI Treatment Approach of BRAF-

Mutated PSCC of the Thyroid 
3 Lam AK, Basnet A, Pandita A, Fullmer J, Sivapiragasam A, Ko YS, et al. Thyroid squamous cell carcinoma: a unique type of cancer in World Health Organization classification. Case Rep Oncol Med. 2020;2017(1): 43-8.

4 Chavan RN, Chikkala B, Biswas C, Biswas S, Sarkar DK. Primary squamous cell carcinoma of thyroid: a rare entity. Case Rep Pathol. 2015;2015:1-3.

5 Tunio MA, Al Asiri M, Fagih M, Akasha R. Primary squamous cell carcinoma of thyroid: a case report and review of literature. Head Neck Oncol. 2012;4(1):8.

6 Struller F, Senne M, Falch C, Kirschniak A, Konigsrainer A, Muller S. Primary squamous cell carcinoma of the thyroid: case report and systematic review of the literature. Int J Surg Case Rep. 2017;37:36-40.

7 Cho JK, Woo SH, Park J, Kim MJ, Jeong HS. Primary squamous cell carcinomas in the thyroid gland: an individual participant data meta-analysis. Cancer Med. 2014 Oct 1;3(5): 1396-403.

8 Tallini G, de Biase D, Repaci A, Visani M. What's new in thyroid tumor classification, the 2017 World Health Organization classification of tumours of endocrine organs. Thyroid FNA Cytol. 2019:37-47.

9 Choi S, Shugard E, Khanafshar E, Quivey JM, Garsa AA, Yom SS. Association between BRAF V600E mutation and decreased survival in patients locoregionally irradiated for anaplastic thyroid carcinoma. Int J Radiation Oncol Biol Phys. 2016 Oct 1;96(2): E356.

10 Nikiforova MN, Kimura ET, Gandhi M, Biddinger PW, Knauf JA, Basolo F, et al. BRAF mutations in thyroid tumors are restricted to papillary carcinomas and anaplastic or poorly differentiated carcinomas arising from papillary carcinomas. J Clin Endocrinol Metab. 2003 Nov;88(11):5399-404.
11 Latteyer S, Tiedje V, König K, Ting S, Heukamp LC, Meder L, et al. Targeted next-generation sequencing for TP53, RAS, BRAF, ALK and NF1 mutations in anaplastic thyroid cancer. Endocrine. 2016 Dec 1;54(3): $733-41$.

12 Subbiah V, Kreitman RJ, Wainberg ZA, Cho JY, Schellens JHM, Soria JC, et al. Dabrafenib and trametinib treatment in patients with locally advanced or metastatic BRAF V600-mutant anaplastic thyroid cancer. J Clin Oncol. 2018 Jan 1;36(1):7-13.

13 Robert C, Grob JJ, Stroyakovskiy D, Karaszewska B, Hauschild A, Levchenko E, et al. Fiveyear outcomes with dabrafenib plus trametinib in metastatic melanoma. $\mathrm{N}$ Engl J Med. 2019 Aug 15;381(7):626-36.

14 Torrez M, Braunberger RC, Yilmaz E, Agarwal S. Primary squamous cell carcinoma of thyroid with a novel BRAF mutation and High PDL-1 expression: a case report with treatment implications and review of literature. Pathol Res Pract. 2020 Oct 1;216(10): 153146.

15 Cabanillas ME, Ferrarotto R, Garden AS, Ahmed S, Busaidy NL, Dadu R, et al. Neoadjuvant BRAF- and immune-directed therapy for anaplastic thyroid carcinoma. Thyroid. 2018 Jul 1;28(7):945-51.

16 Ko YS, Hwang TS, Han HS, Lim SD, Kim WS, Oh SY. Primary pure squamous cell carcinoma of the thyroid: report and histogenic consideration of a case involving a BRAF mutation. Pathol Int. 2012;62(1):43-8.

17 Rausch T, Benhattar J, Sutter M, AndrejevicBlant S. Thyroid carcinoma with papillary and squamous features: report of a case with histogenetic considerations. Pathol Res Pract. 2010 Apr 15;206(4):263-9.

18 Acosta AM, Pins MR. Papillary thyroid carcinoma with extensive squamous dedifferentiation metastatic to the lung: BRAF mutational analysis as a useful tool to rule out tumor to tumor metastasis. Virchows Arch. 2016 Feb 1; 468(2):239-42.
19 Rupani A, Hughes OR, Watkinson J, Nutting CM, Thway K. Metastatic papillary thyroid carcinoma with adjacent BRAF (V600E)-mutated squamous cell carcinoma. Int J Surg Pathol. 2017 May 21;25(3):243-5.

20 Lee JI, Chung YJ, Lee SY. Papillary thyroid carcinoma recurring as squamous cell carcinoma 10 years after total thyroidectomy: lessons from rapidly progressive papillary thyroid carcinoma. Intern Med. 2013 Jul 15; 52(14):1593-7.

21 Montero-Conde C, Ruiz-Llorente S, Dominguez JM, Knauf JA, Viale A, Sherman EJ, et al. Relief of feedback inhibition of HER3 transcription by RAF and MEK inhibitors attenuates their antitumor effects in BRAF-mutant thyroid carcinomas. Cancer Discov. 2013 May;3(5):520-33.

22 Wagle N, Emery C, Berger MF, Davis MJ, Sawyer A, Pochanard P, et al. Dissecting therapeutic resistance to RAF inhibition in melanoma by tumor genomic profiling. J Clin Oncol. 2011 Aug 1 [cited 2021 May 19];29(22):3085-96.

23 Chintakuntlawar AV, Rumilla KM, Smith CY, Jenkins SM, Foote RL, Kasperbauer JL, et al. Expression of PD-1 and PD-L1 in anaplastic thyroid cancer patients treated with multimodal therapy: results from a retrospective study. J Clin Endocrinol Metab. 2017 Jun 1; 102(6):1943-50.

24 Bastman JJ, Serracino HS, Zhu Y, Koenig MR, Mateescu V, Sams SB, et al. Tumor-infiltrating T Cells and the PD-1 checkpoint pathway in advanced differentiated and anaplastic thyroid cancer. J Clin Endocrinol Metab. 2016 Jul 1;101(7):2863-73.

25 Capdevila J, Wirth LJ, Ernst T, Ponce Aix S, Lin C-C, Ramlau R, et al. PD-1 blockade in anaplastic thyroid carcinoma. J Clin Oncol. 2020 May 4;38(23):2620-7. 